\title{
A Pattern Recognition Approach for Identification of Transducer-Structure Debonding using Lamb Waves
}

\author{
Durai Arun P, C.N. Sathyanarayana, S. Raja, and VPS Naidu \\ CSIR-National Aerospace Laboratories \\ Bangalore, India
}

\begin{abstract}
In structural health monitoring, using piezoelectric transducers to generate high frequency elastic waves like Lamb waves in the structures is eminent. In general, piezoelectric transducers are assumed to be perfectly bonded with the host structure; however, in practical environment, there are possibilities for them to have faults. Since detecting, locating and assessing damages in a structure depend solely on transducer responses, transducer fault identification is vital. By using electrical admittance, axial strain and shear stress as function of frequencies or in analog interface circuits to identify faulty transducers, lead to demand of circuitry and processes, consequently increasing the implementation complexity. Hence, we propose a pattern recognition system that can identify transducers that are partially bonded to host structure. This pattern recognition system employs classification for features extracted from instantaneous Lamb wave signals with no need of baseline data.
\end{abstract}

Keywords-Lamb Waves, Sensor Debonding Identification, Structural health monitoring, PZT

\section{INTRODUCTION}

Structural sensing, structural health monitoring, structural performance assessment, and health prognosis are basic components of modern structural engineering practice [1]. A system that detects damages, locates them, estimates their severity and able to give a structure's lifetime, is a Structural Health Monitoring System [2]. The most common NDE technique for damage detection and localization being researched is using elastic waves to extract structural integrity information. When elastic waves propagate through a structure they undergo changes, if there are any structural anomalies. Extracting those anomaly-sensitive changes from elastic waves is the complexity involved in any SHM system design [3]. Among different types of elastic waves, Lamb wave is used in researches due to its advantages. Lamb waves can propagate over long distances through a material medium at higher velocities and it is sensitive to structural abnormalities and inhomogeneity, near its propagation path. Lamb wave generation and sensing relies on piezoelectric transducers such as Lead Zirconate Titanate (PZT) [4]. Epoxy adhesive bonds piezoelectric transducers on the host structure and for the actuation or sensing, transducers' ohmic contacts are connected to any signal source. Often these transducers are assumed to have no faults; while in a practical environment, these do have the possibilities of having faults. For example,

Financial support granted by Science \& Engineering Research Board, Department of Science \& Technology, Govt. of India. these transducers are assumed to be perfectly bonded to the host structure, but they have the possibility of having partial bonding. Identifying faulty transducer in a SHM system is important, for damage detection and localization, depends on transducers' responses. Currently, methods used in practice and research for transducer fault identification in PZTs are based on Impedance, Electrical admittance, Axial Strain, and Shear Stress as a function of frequencies or analog interface circuit, to compare extracted parameters with prior information available [5-11]. Other approaches use data processing techniques like Principal Component Analysis (PCA), Minimum-Mean Square Error (MMSE) to identify faulty transducers with the help of reference i.e. either by having prior data or generating instantaneous data. All these approaches demand either more circuitry or processes, increasing the complexity in implementation, when a large number of transducers are involved. Thus, we propose a pattern recognition system that identifies faulty transducers from healthy transducers. The type of fault we chose to work is the transducer-host structure interface debonding. Features extracted from instantaneous Lamb wave signals are used in the proposed classifier requiring no reference or baseline data.

\section{Methodology}

\section{A. Lamb Wave Generation and Signal Conditioning}

Lamb wave propagation is of two modes, namely, symmetric $\left(\mathrm{S}_{0}\right)$ and anti-symmetric $\left(\mathrm{A}_{0}\right)$ mode. The determination of excitation frequency and mode for Lamb Wave generation is decided by mode dispersion curves of the structure under inspection [4]. In the present work, $A_{0}$ Lamb wave mode is generated using two PZTs placed in the centre of the structure, i.e. aluminium plate and eight PZTs are bonded as a circular array of transducers. A n-cycle sine tone burst narrow banded by Hanning window is fed as input to the top transducer in the centre of the structure, which acts as an emitter. The inverse of the narrow banded sine tone burst is fed to the bottom transducer located at the centre of the structure. The simultaneous inverse voltage variation of transducers enables them to generate pure $A_{0}$ Lamb waves in the structure. In the proposed system, moving average smoothing filter is used to reduce noise and unnecessary spikes, whose window span size is determined by the sampling frequency of the data acquisition system employed. 


\section{B. Signal Segmentation}

Transducer configuration is vital in Lamb wave based damage detection techniques. Since Lamb waves travel at higher velocities, along with the transmitted wave, the reflections from the boundaries of structure are also sensed. In the proposed system, Hilbert transform is used for segmenting intended signals from the total length of all transducers' sensed signals. The envelopes of all transducers' sensed signals are obtained by performing the modulus operation after applying Hilbert transform to all acquired signals and averaged. To that averaged-envelope, peak detection technique is used and the first peak detected is identified as the segmentation reference time/sample of intended wavelet signal. Based on the excitation frequency and sampling frequency, the segmentation-size in terms of number of samples, is determined. The segmentation window is calculated by segmentation reference time/sample and segmentation-size in terms of number of samples. Let ' $\mathrm{x}$ ' be a transducer sensed signal in total acquisition length, ' $\mathrm{n}$ ' be the sample index, 'Sseg' be the segmentation size, 'npeak' be the segmentation reference time/sample and ' $y$ ' be the segmented signal with ' $n_{\text {seg' }}$ being its sample index, then the equation (1) shows the relation between full length signal and segmented signal.

$$
y\left(n_{\text {seg }}\right)=\left\{x(n) \mid n_{\text {peak }}-\left(\frac{s_{s e g}}{2}\right) \leq n \leq n_{\text {peak }}+\left(\frac{s_{s e g}}{2}\right)\right\}_{(1)}
$$

By applying this segmentation window to emitter and receiving transducer responses, only the needed wavelet signals are segmented. Further analysis and processes are performed on those segmented signals only.

\section{Feature Extraction and Feature Space Formation}

For Lamb wave signals, time domain analysis, frequency domain analysis, and joint time-frequency domain analysis are carried out to extract information from them. In this work, we extract descriptive statistics, wave intensity, and time of flight as features to identify transducers whose interface has been debonded. For calculating the time of flight, both Hilbert transform based envelopes, and continuous wavelet transform based square averaged power spectrum (SAP) was used and found that, there is no difference between the methods in value. Equation (2) shows the formula for calculating the time of flight $(\mathrm{ToF})$, where ' $i$ ' is the number of transducer and curve may be an envelope or SAP.

Time of Flight $=$ Time $\mid \max \left(i \quad \begin{array}{r}\text { th } \\ \text { receiver curve })\end{array}\right.$

For extracting wave intensity information, transmission coefficients of all receiving transducers are calculated. Transmission coefficient is the ratio of the largest magnitude of the intended wavelet after the wave propagating across the structure, captured by the transducer, to that of the incident intended wavelet. Similar to the time of flight calculation, to calculate the transmission coefficient, both Hilbert transform based envelopes, and continuous wavelet transform based square averaged power spectrum (SAP) was used and no difference in values based on the method of calculation was found. Equation (3) shows the formula to calculate transmission coefficients of all transducers.

Trans_Coeffi $=\max ($ receiveri curve $) / \max ($ emitter curve)

Standard deviation and autocorrelation of segmented transducer responses were calculated to extract descriptive statistics, since other statistical measures did not depict the significant changes relevant to the interface-debonding identification. Equations (4) \& (5) show the formulae to calculate Standard deviation and autocorrelation (R) respectively where, $\mathrm{i}=1$ to total no. of transducers.

$$
\begin{aligned}
& \text { Standard deviation } i=\sqrt{\sum_{j=1}^{m} \frac{\left(x_{j}-\bar{x}\right)^{2}}{m}} \\
& R_{i}=\max \left(\frac{1}{N-m} \sum_{j=0}^{j-m-1} x(j) * x(j+m)\right)
\end{aligned}
$$

Although the above-mentioned features when projected in 1-D feature space indicate transducer-structure interface debonding, formulating a generalized classification rule has become a complicated task. To overcome this difficulty, feature space of 2-D was experimented [12]. All possible combinations of feature space with time of flight as its $\mathrm{x}$-axis and other features as y-axis were compared to each other. In comparison, it was observed that all features spaces showed similar clustering of the features after applying the logarithm to the features and adding bias to the feature vectors. Fig. 1 shows all feature space combinations of the dataset having both fully bonded transducers and partially bonded transducers. Since the proposed system will be extended to other functionalities like damage detection and localization, continuous wavelet transform will be used. Thus, the feature space of Time of flight vs. peak amplitude of square averaged wavelet power spectrum (SAP) is chosen for transducerstructure interface debonding identification. Let 'CWT' be the continuous wavelet transform of a signal ' $x(n)$ ', ' $M$ ' be the number of frequency scales, and 'SAP(n)' be the Square averaged wavelet power spectrum. Equation (6) shows the formula to calculate the SAP curve from wavelet transformation.

$$
\operatorname{SAP}_{i}(n)=\frac{1}{M} \sum_{k=1}^{M}\left|C W T\left(a_{k}, n\right)\right|^{2}
$$

\section{Classification}

Feature space generated from the segmented transducer responses using equations (2) and (6) has shown different data clusters characterizing fully bonded transducers from partially bonded transducers. From analysis [13-15], it is found that in the feature space, fully bonded transducers' data form a single cluster and debonded transducers' data have clustered in 
accord with their type of interface debonding. If all transducers are fully bonded to the host structure, the classifier should not misclassify the data, resulting in false negative error. Thus, this system has the complexity of identifying single cluster. In data clustering techniques like k-means clustering detecting the presence of a single cluster is the most difficult problem to solve. However, for this classification problem, the inter class distances of features are significantly higher, paving way for dispersion metrics to aid in identifying single cluster. When there are multi clusters, i.e. transducerstructure interface undergoes debonding, using suitable decision boundary lines, debonded transducers can be identified along with its type. Let 'i' be the index of transducers (here, $\mathrm{i}=1$ to 8 ), ' $\mathrm{N}$ ' be the total number of transducers, ' $\mathrm{T}$ ' be the Time of flight and 'A' be the Peak amplitude, both calculated from the SAP of the continuous wavelet transform of the segmented transducer responses. The steps involved for classification are,

1. Assigning calculated features as feature vector $\mathrm{F}=\left[\mathrm{T}_{\mathrm{i}}, \mathrm{A}_{\mathrm{i}}\right]$

2. Calculating the Range $R$ from feature vector $F, R=\left[R^{T}, R^{A}\right]$

3. If $\mathrm{R}$ is less than Cluster-Threshold

a. Only Single Cluster is present in the dataset, hence Class $=1$

4. If $\mathrm{R}$ is greater than Cluster-Threshold

Loop $\mathrm{i}=1$ to $\mathrm{N}$

If $\mathrm{F}_{\mathrm{i}}>=$ Debond-Threshold

Transducer-Classi = 'Fully Bonded'

Else If $\mathrm{F}_{\mathrm{i}}>=$ Debond-Type-Threshold

Transducer-Classi $=$ 'Debonded Bonded'

End

End Loop

\section{EXPERIMENTS AND RESULTS}

\section{A. Experiment Setup}

Two aluminium plates having dimensions of 500x500x1.5 $\mathrm{mm}^{3}$ are placed in between two frames and mounted on the fixture using M6 bolts to simulate C-C-C-C boundary condition and surface mounted with ten Lead zirconate titanate (PZT) transducers using standard epoxy adhesive (Araldite) and cured as per the standard guidelines. Out of ten PZT transducers on a plate, two are emitters designated, as E1 \& E2 and another eight are receivers designated as R1 to R8. The dimensions of the emitting transducers and the receiving transducers are $15 \times 15 \times 0.5 \mathrm{~mm}^{3}$ and $10 \times 10 \times 0.5 \mathrm{~mm}^{3}$ respectively. In both plates, two emitting transducers are placed in the centre of the plates and all other transducers are bonded in a circular array defined by pitch circle of $200 \mathrm{~mm}$ diameter. Those receiving transducers have an angular spacing of $45^{\circ}$ with each other from the pitch circle centre. In plate 1 , E1 \& E2 transducers and R1 to R8 transducers are perfectly bonded to the structure. In plate 2, E1 \& E2 transducers and few of receiving transducers are perfectly bonded. Three types of $50 \%$ transducer-structure interface-debonding are introduced in other transducers in plate 2, namely, type-A, type-B, and type-C. Simulated natures of debonding of sensors are tabulated in Table 1 and schematically shown in Fig. 2.
TABLE I. TyPES \& NATURE OF DEBONDING SIMULATED IN PZT TRANSDUCERS

\begin{tabular}{|c|l|l|}
\hline Type & \multicolumn{1}{|c|}{ Nature } & \multicolumn{1}{c|}{ Changes in Wave Propagation } \\
\hline A & Outside PCD & $\begin{array}{l}\text { Waves faces fully bonded area of PZT first } \\
\text { then debonded area }\end{array}$ \\
\hline B & Inside PCD & $\begin{array}{l}\text { Waves faces debonded area of PZT first then } \\
\text { fully bonded area }\end{array}$ \\
\hline C & Across PCD & $\begin{array}{l}\text { Waves faces both fully bonded \& debonded } \\
\text { area where boundary of bonding interface is } \\
\text { perpendicular to loci of Pitch Circle. }\end{array}$ \\
\hline
\end{tabular}

\section{B. Lamb Wave Generation and Sensing}

A $35 \mathrm{KHz} \mathrm{A}_{0}$ Lamb Wave mode narrow banded by Hanning window are generated by applying out of phase 3.5 cycled sine tone burst with $20 \mathrm{~V}$ peak to peak voltage on E1 \& E2 by Tektronix Dual Channel Arbitrary Function Generator (AFG3022B). The propagated waves are sensed by $\mathrm{R} 1$ to $\mathrm{R} 8$ through Tektronix Digital Storage Oscilloscopes (TDS2014B). The Function Generator and Oscilloscopes are interfaced to PC by TekVISA and MATLAB software. Signals are processed after 16 times linearly averaged acquisition and record length is 2500 samples.

\section{Transducer-Structure Interface Debonding Identification by Pattern Classification}

Inside MATLAB environment, all channel data are smoothened by moving average filter whose window span is 91 samples. As mentioned in the Methodology section, Hilbert transforms and modulus operation is applied to all smoothened data to obtain their envelopes. Then these envelopes are averaged and peak detection is employed to find the segmentation reference time/sample. With an excitation frequency of $35 \mathrm{KHz}$ and 10 times its sampling frequency, the segmentation-size is determined to be 560 by trial \& error method. Now with these parameters, the intended actuation signal and all the received signals are segmented. The continuous wavelet transform is applied to all segmented data, giving time-frequency distribution as a 2-dimensional array, and from that 2D array square averaged power spectrum (SAP) for all segmented data is calculated. Peak detection is applied to all SAP curves to calculate the Time of Flight and to find Peak Wavelet Power Coefficient corresponding to reference time/sample. Both Time of Flight and Peak Wavelet Power Coefficient are assigned as feature vectors. Feature space of Plate 1 depicting single cluster formation representing that all transducers are perfectly bonded to the structure. Multi cluster formations for Plate 2 representing few transducers are perfectly bonded to the structure and some are not. Classification rule is applied on both feature sets, and transducers with partial debonding are identified among the transducers, which are perfectly bonded. Fig. 3 shows the feature extraction process and classified feature space of both plates.

\section{CONCLUSION AND FUTURE WORK}

Transducer fault diagnosis is important in a structural health monitoring system, since its architecture relies on transducer's responses. Among different types of faults 
existing, transducer-structure interface debonding is the most common fault that can occur in piezoelectric transducers like PZT. A0 mode Lamb waves are generated at the centre of the aluminium plates and other transducers are placed in the circular array configuration to sense them. Three types of $50 \%$ debonding are experimented for pattern recognition based transducer-structure interface debonding identification. Time of flight and wavelet power coefficient, both obtained from the square averaged power spectrum by continuous wavelet transform of Lamb Wave signals. Features of transducers, which are perfectly bonded, formed a cluster and features of transducers, which are partially, bonded grouped into clusters representing their type of debonding. If all transducers are perfectly bonded, then the features form a single cluster in feature space and when feature space contains features of both perfectly bonded transducers and partially bonded transducers multi clusters are formed. Data dispersion and decision boundary lines are used for classification. Thus, a pattern recognition system that identifies transducer-structure interface debonding without any prior knowledge of debonding is proposed. Still, the proposed system is limited by the geometry of the structure, transducers' location and configuration. Designing a classification system, which is not constrained by the geometry to identify transducer-structure interface debonding, is the future work.

\section{ACKNOWLEDGMENT}

This work is carried out under a project funded by Science \& Research Board, Department of Science \& Technology, Govt. of India is gratefully appreciated.

\section{REFERENCES}

[1] D Huston, Structural Sensing, Health Monitoring, and Performance Evaluation, CRC Press, 2011.

[2] Victor Giurgiutiu, Structural Health Monitoring with Piezoelectric wafer

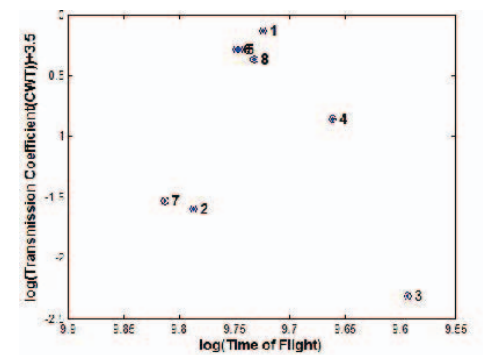

a. ToF vs Peak Amplitude of Wavelet based SAP curve

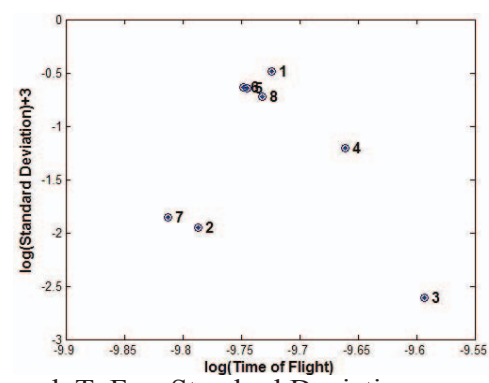

d. ToF vs Standard Deviation of Transducer Responses

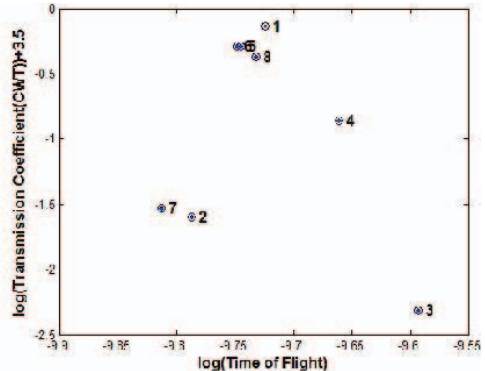

b. ToF vs Wavelet based Transmission Coefficient

Fig. 1 Feature space combinations of dataset having both fully bonded transducers \& partially bonded transducers

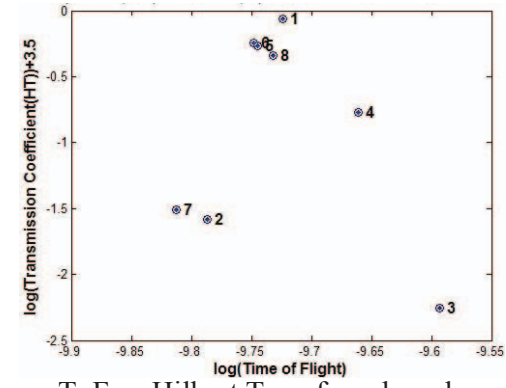

c. ToF vs Hilbert Transform based Transmission Coefficient

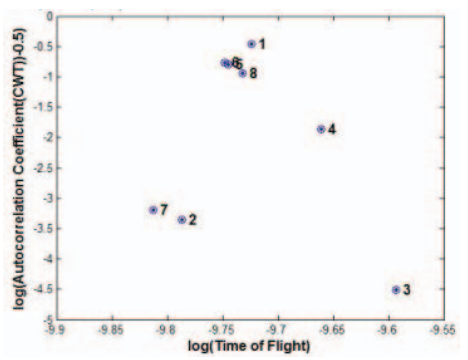

e. ToF vs Maximum Autocorrelation Coefficient of Transducer Responses 


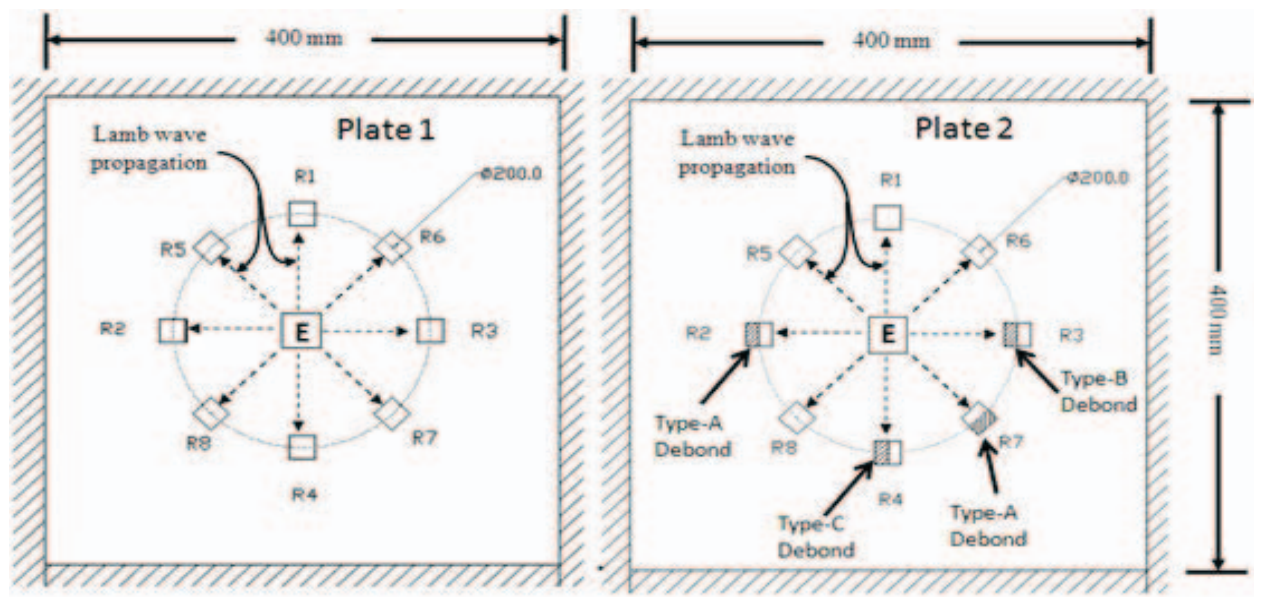

Fig. 2 Schematic Representations of Aluminium Plates with Emitting \& Receiving Transducers

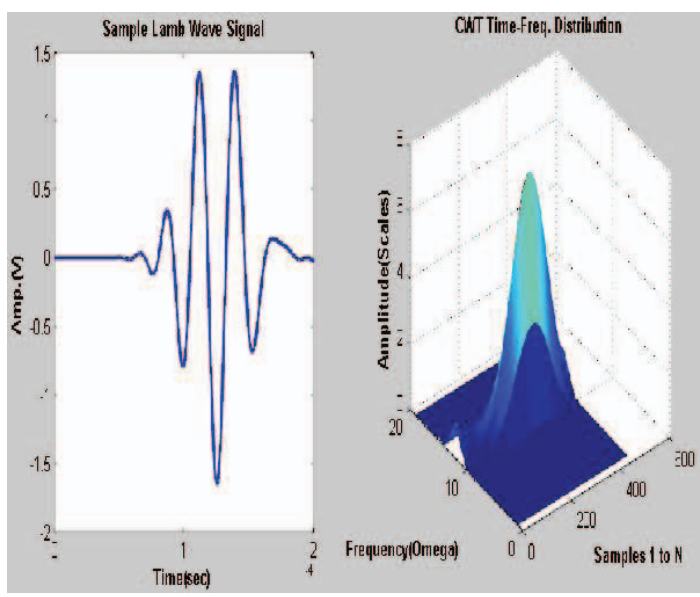

a. SAP Extraction from Time-Frequency Distribution of a Transducer's Lamb Wave Signal

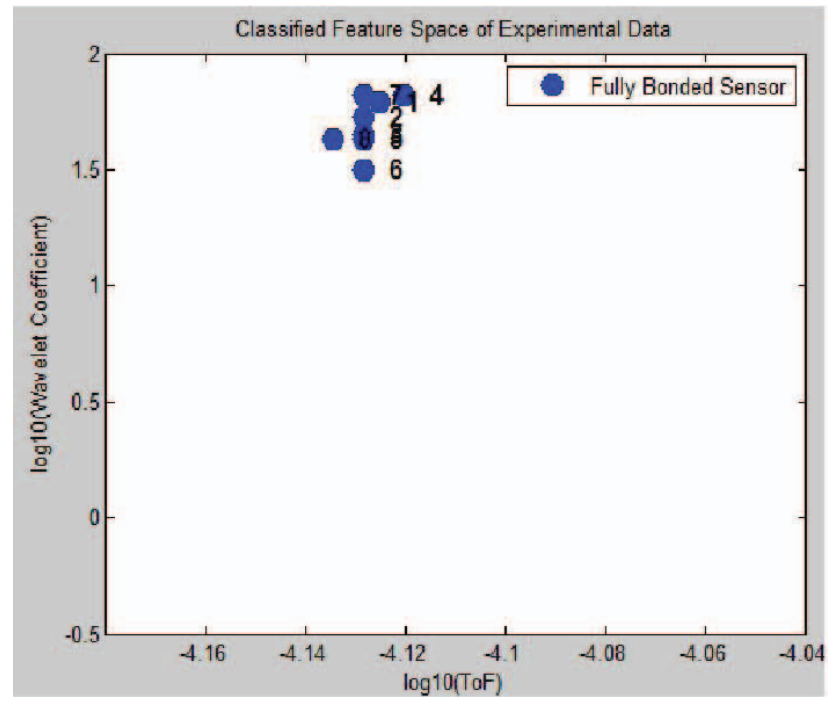

c. Classified Feature Space of All Transducers Perfectly Bonded
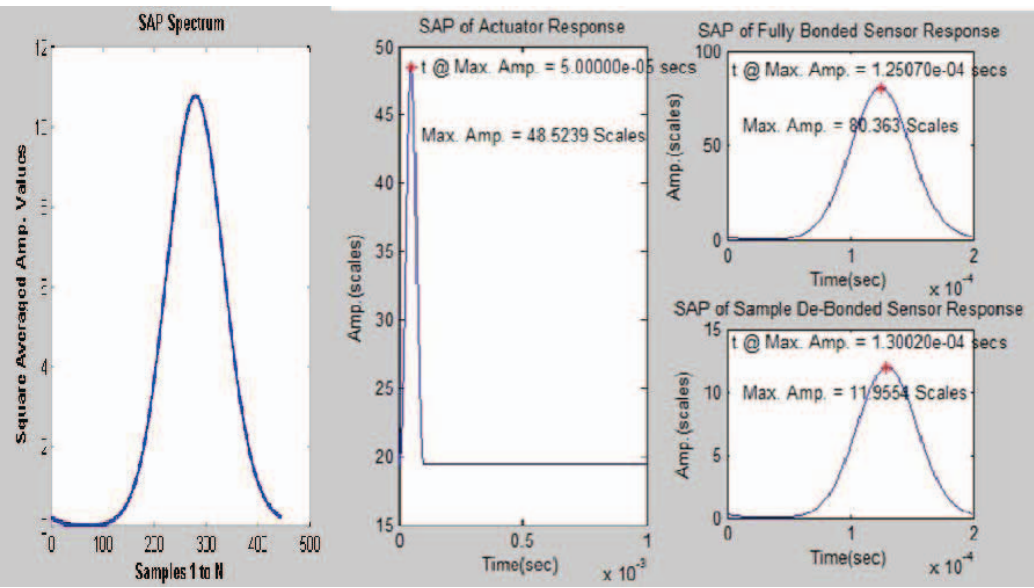

b. Time of Flight \& Wavelet Power Coefficient Extraction from SAP curve

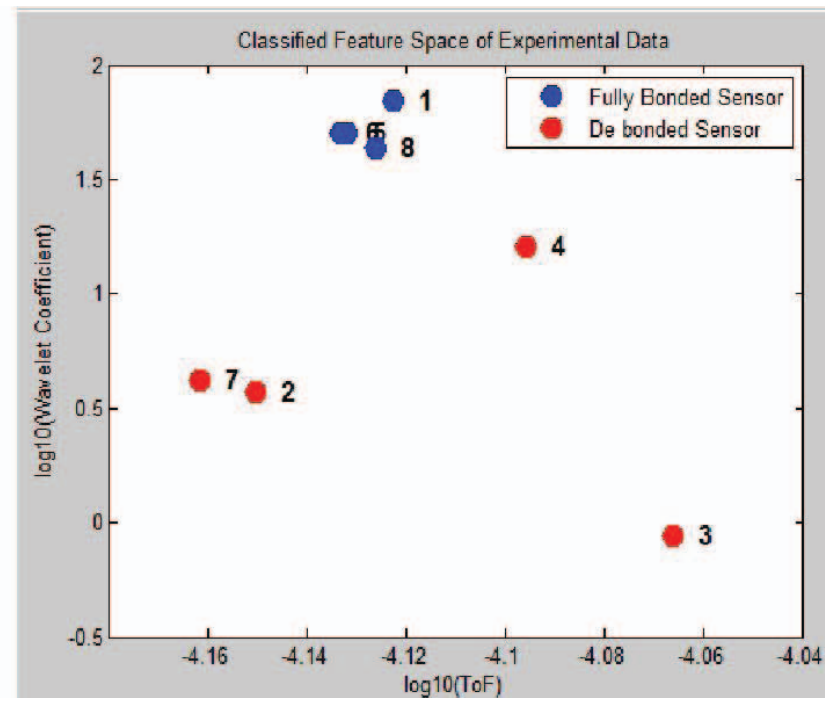

d. Classified Feature Space of Transducers Perfectly Bonded and Partially Bonded (Debonded)

Fig. 3 Time of Flight \& Wavelet Power Coefficient Extraction and Pattern Classification 